\title{
Polished rice as natural sources of cancer-preventing geranylgeranoic acid
}

\author{
Takashi Muraguchi, Kyoko Okamoto, Maiko Mitake, Hiroko Ogawa and Yoshihiro Shidoji* \\ Molecular and Cellular Biology, Graduate School of Human Health Sciences, Siebold University of Nagasaki, Nagayo, Nagasaki 851-2195, Japan
}

(Received 23 August, 2010; Accepted 28 September, 2010; Published online 26 April, 2011)

\begin{abstract}
Geranylgeranoic acid, a 20-carbon polyprenoic acid (all-trans 3,7,11,15-tetramethyl-2,4,6,10,14-hexadecatetraenoic acid) and its derivatives were previously developed as synthetic "acyclic retinoids" for cancer chemoprevention. Recently, we demonstrated the natural occurrence of geranylgeranoic acid in various medicinal herbs (Shidoji and Ogawa, 2004). In this present study, we present several lines of evidence to demonstrate that geranylgeranyl diphosphate taken in foods could be metabolized to GGA through geranylgeraniol and geranylgeranyl aldehyde via the following steps: 1) The conversion from geranylgeranyl diphosphate to geranylgeraniol was demonstrated to occur by the action of bovine intestinal alkaline phosphatase, with a $K_{\mathrm{m}}$ of $46.1 \mu \mathrm{M}$. 2) Geranylgeraniol oxidase-mediated conversion of geranylgeraniol to geranylgeranyl aldehyde was revealed in rat liver homogenates, which activity was mainly localized in the mitochondrial fraction. The mitochondrial enzyme showed a $K_{\mathrm{m}}$ of $92.9 \mu \mathrm{M}$. 3) The conversion of geranylgeranyl aldehyde to geranylgeranoic acid by geranylgeranyl aldehyde dehydrogenase in rat liver homogenates was absolutely dependent on exogenously added NAD+ ${ }^{+}$or NADP+. The $K_{\mathrm{m}}$ of the mitochondrial geranylgeranyl aldehyde dehydrogenase was $27.5 \mu \mathrm{M}$ for geranylgeranyl aldehyde. Taken together, our data suggest that cancer preventive geranylgeranoic acid could be a physiological metabolite from commonly consumed foods.
\end{abstract}

Key Words: geranylgeranyl diphosphate, geranylgeraniol, geranylgeranoic acid, cancer chemoprevention, polished rice

G eranylgeranoic acid (GGA), a 20-carbon polyprenoic acid (alltrans 3,7,11,15-tetramethyl-2,4,6,10,14-hexadecapentaenoic acid) consisting of 4 isoprene units and its derivatives were historically developed as synthetic "acyclic retinoids" by screening for their ability to bind to cellular retinoic acid-binding protein ${ }^{(1)}$ as well as to nuclear retinoid receptors, ${ }^{(2)}$ which exert transcriptional activation of certain hepatocyte-specific genes in hepatoma cells. ${ }^{(3)}$ 4,5-Didehydro GGA, one of the most potent acyclic retinoids, has been reported to prevent chemically induced and spontaneous hepatocarcinogenesis in animals. ${ }^{(4,5)}$ Further, the efficacy of 4,5-didehydro GGA in preventing second primary hepatoma was proven in a placebo-controlled double-blinded and randomized phase II clinical trial using postoperative hepatoma patients, with few side effects observed. ${ }^{(6)}$ Later, it was revealed that the oral administration of the polyprenoic acid for 12 months significantly increased the 5-year survival rate in these patients after a radical therapy for primary hepatoma. ${ }^{(7)}$

Although GGA, an acyclic retinoid, shares characteristics of natural cyclic retinoids in vitro, GGA also apparently differs from natural retinoids such as all-trans and 9-cis retinoic acids in the following respects: 1) GGA up-regulated albumin mRNA expression in human hepatoma-derived cell lines, HuH-7 and PLC/PRF-5, whereas all-trans retinoic acid down-regulated it;(3)
2) GGA was much less toxic than natural retinoids in a human hepatoma cell line in the presence of fetal bovine serum (FBS), ${ }^{(4)}$ and 1-yr intake of 4,5-didehydroGGA $(600 \mathrm{mg} /$ day $)$ gave no apparent side effects in the above-mentioned clinical trial;(6,7) 3 ) unlike natural retinoids, GGA showed no growth-promoting activity in vitamin A-deficient animals (unpublished observation); and finally, 4) GGA induced apoptosis in hepatoma cell lines in the absence of FBS, whereas neither all-trans nor 9-cis retinoic acid did so. ${ }^{(8,9)}$ Hence, an important question has arisen as to what kinds of natural compounds other than retinoids are mimicked by acyclic retinoids.

From a chemical structural point of view, GGA belongs to the family of isoprenoids (or terpenoids specially in plants), which form the most chemically diverse family of molecules found in nature and are widely distributed in many different organisms including viruses, bacteria, plants, fungi, yeasts, and mammals. ${ }^{(10,11)}$ The isoprenoid biosynthetic pathway (Fig. 1) is build around a family of diphosphate esters of linear alcohols that contain increasing numbers of isoprene units. Beginning with the $\mathrm{C}_{5}$ molecule DMAPP (dimethylallyl diphosphate), a series of $\mathrm{C}_{10}$ GPP (geranyl diphosphate), $\mathrm{C}_{15}$ FPP (farnesyl diphosphate), $\mathrm{C}_{20}$ GGPP (geranylgeranyl diphosphate), and higher-molecular-weight isoprenoid diphosphates are formed by tail-to-head addition of $\mathrm{C}_{5}$ IPP (isopentenyl diphosphate) to the growing chain. These compounds are the substrates for biosynthesis of all isoprenoid metabolites, including monoterpenes $\left(\mathrm{C}_{10}\right)$, sesquiterpenes $\left(\mathrm{C}_{15}\right)$, diterpenes $\left(\mathrm{C}_{20}\right)$, sterols $\left(\mathrm{C}_{30}\right)$, carotenoids $\left(\mathrm{C}_{40}\right)$, ubiquinones $\left(\mathrm{C}_{50}\right)$, dolichols $\left(\mathrm{C}_{80-100}\right)$, and prenylated proteins. Among these, biologically active isoprenoids are found in insects (juvenile hormone, ecdysone), plants (gibberellic acid, abscissic acid), and fungi/yeasts (prenylated mating hormone)..$^{(12,13)}$ Many plantproduced isoprenoids such as fat-soluble vitamins are essential nutrients in human diets, and some are used as chemotherapeutic agents with antitumor activities, such as taxol. ${ }^{(14)}$

Recent observations have led to the identification of new biologically active compounds that are derived from intermediates of the isoprenoid/cholesterol pathway. ${ }^{(15)}$ These bioactive compounds include certain sterols, oxysterols, farnesol (FOH; $\mathrm{C}_{15}$ ), and geranylgeraniol $\left(\mathrm{GGOH} ; \mathrm{C}_{20}\right) .{ }^{(16)}$ Until we reported on the natural occurrence of cancer-preventing GGA in medicinal herbs, ${ }^{(17)}$ GGA had long been recognized only as a synthetic chemical developed for cancer-chemoprevention. Based upon its chemical structure, we reasonably assumed that GGA could be enzymatically derived from GGOH, which is a common precursor of all natural diterpenoids hydrolyzed from GGPP by a specific pyrophosphatase enzyme (Fig. 1). GGPP is one of the key isoprenoid intermediates to be allocated to the synthesis of various end products necessary for plant growth and defense. The dis-

To whom correspondence should be addressed. E-mail: shidoji@sun.ac.jp 


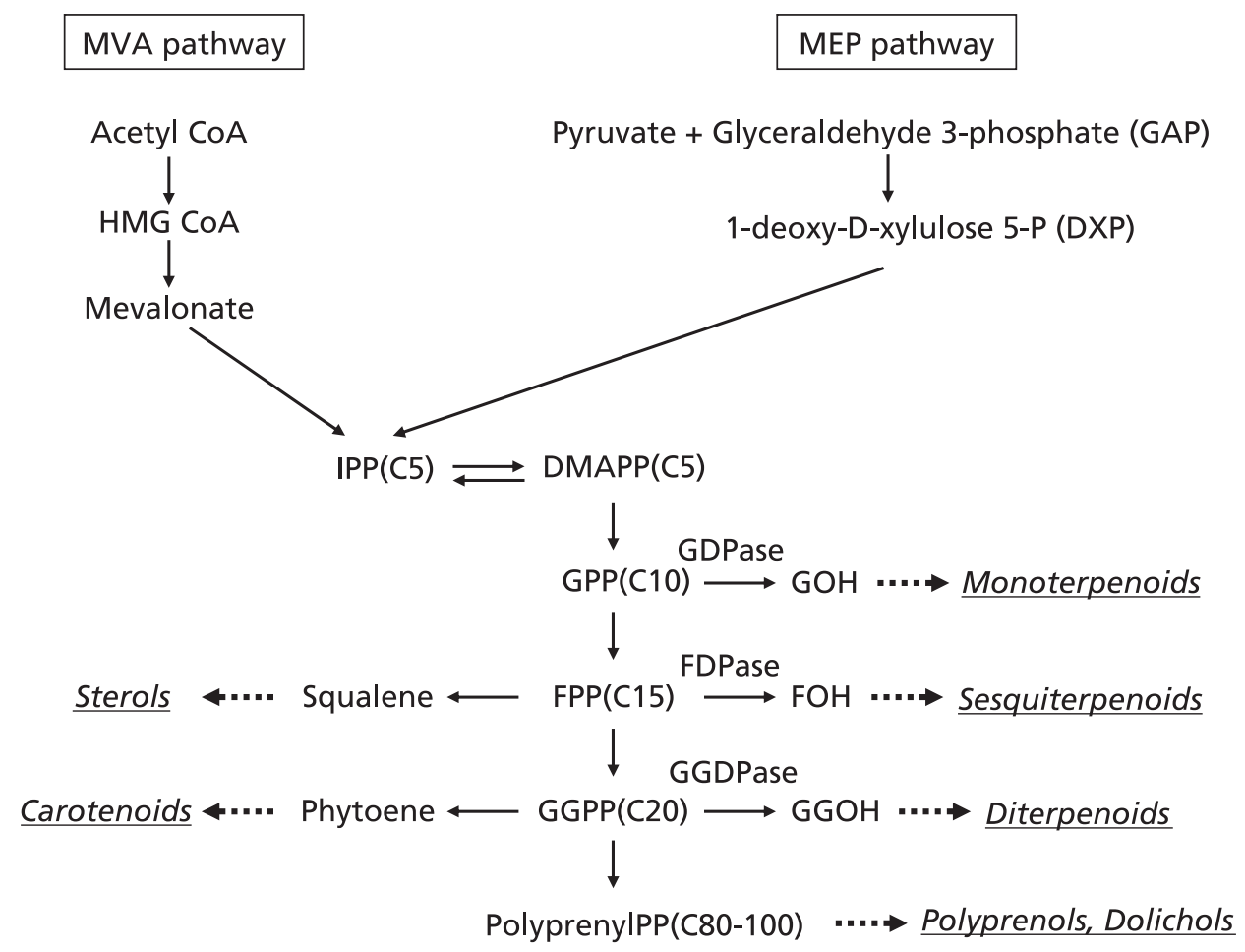

Fig. 1. Schematic diagram of the isoprenoid production. Pathways via acetate/mevalonate (MVA) or GAP/pyruvate [methylerythritol phosphate (MEP)] are shown. The details are described in the text.

covery of natural GGA in herbs led us to search for possibly earlier precursors as the exogenous source of GGA. The idea of producing $\beta$-carotene in "Golden Rice" (see below) gave us a hint to solve this quest.

Rice (Oryza sativa) is a global food staple and is eaten often in high amounts in Asian countries including Japan. Rice is usually processed to remove its outer layers (pericarp, tegmen, and aleurone layers) to leave the rice endosperm (rice grain) for longterm storage and use, especially in tropical and subtropical areas. The oil-rich aleurone layer is removed so that the rice will not become rancid during storage. ${ }^{(18)}$ As a result, the edible part of rice grains consists of the endosperm, filled with starch granules and protein bodies, but it lacks several essential nutrients for the maintenance of health, such as carotenoids, which exhibit provitamin A-activity.

Only scanty information is available on the nutritional effects of lipids in the rice endosperm, though polyunsaturated fatty acids are abundant in the aleurone layer, which is usually removed. In 1997, Burkhardt et al. ${ }^{(19)}$ suggested the presence of GGPP in the immature rice endosperm. GGPP is a precursor essential for the biosynthesis of $\beta$-carotene, but rice naturally does not have the metabolic pathway to make $\beta$-carotene in the endosperm. To convert GGPP to $\beta$-carotene, 4 additional plant enzymes were needed: phytoene synthase, phytoene desaturase, $\beta$-carotene desaturase, and lycopene $\beta$-cyclase. These enzymes were identified and their genes were isolated from various plants and bacteria. In 2000, Ye et $a l^{\left({ }^{(18)}\right.}$ put all this information together. They established the entire $\beta$-carotene biosynthesis pathway in rice endosperm by using Agrobacterium for gene delivery. By 2002, Beyer et al. ${ }^{(20)}$ had refined the technique and were able to transform rice with genes from daffodil and bacteria to produce rice having the $\beta$-carotene pathway, which they termed "Golden Rice". The first transgene condenses 2 molecules of GGPP in a tail-to-tail manner into phytoene (Fig. 1). Since no exogenous substrate is supplied, the transgenic crop has no choice but to pick up an endogenous substrate to produce $\beta$-carotene, indicating that Golden Rice may increase nutritional value by enzymatically changing endogenous lipids and that an appreciable amount of GGPP, a substrate for phytoene synthase, should exist in wild-type rice endosperm.

Accordingly, we studied our hypothesis that GGPP in rice could be a source of GGA for us. Dietary GGPP could be readily dephosphorylated to GGOH by intestinal alkaline phosphatase (ALP) within the lumen of the small intestine. Intestinal ALP, mostly bound to the brush-border membrane of the absorptive cells, serves as an ectoenzyme facing the luminal space. ${ }^{(21)}$ Then GGOH may be absorbed across the cell membrane and into the enterocytes and conveyed into hepatocytes via the bloodstream.

In the present study, we confirmed the presence of GGPP and its derivatives in some cultivars of rice commercially available in Japan. To answer the question as to whether GGPP in rice could be converted into cancer-preventing GGA by animal enzymes, in this present study we demonstrated the enzymatic conversion from GGPP to GGOH by bovine intestinal ALP and also from GGOH to GGA through GGal by rat liver mitochondrial enzymes in the presence of $\mathrm{NAD}^{+}$or $\mathrm{NADP}^{+}$.

\section{Materials and Methods}

Materials. All-trans GGA, all-trans GGOH, and all-trans GGal were prepared by Kuraray Co. (Okayama, Japan). GGPP was purchased from Sigma-Aldrich (St. Louis, MO). Methanol, n-hexane, chloroform (HPLC grade), ethanol, dehydrated diethylether, $5 \mathrm{M}$ hydrogen chloride $(\mathrm{HCl})$ solution, and $5 \mathrm{M}$ sodium hydroxide $(\mathrm{NaOH})$ solution were from Wako Pure Chemical Industries (Osaka, Japan). Ammonium acetate came from Fluka Bio Chemika (Switzerland); and N-methyl-N-nitro-N-nitrosoguanidine, from GL Sciences (Tokyo, Japan). All other chemicals were of reagent grade.

Extraction of lipids from rice. Four cultivars of Japonica 
rice (Oryza sativa L.), Koshihikari, Kinuhikari, Hinohikari and Karinomai, were surveyed, which were generously donated by Mr. Toshiyuki Sada, Varieties and Seeds Section, Field Crops Division, Nagasaki Agricultural and Forestry Experiment Station (Nagasaki, Japan). All of them were harvested in 2000 and stored dry at room temperature until used.

Rice was homogenized in methanol with Polytron-MR3100 (probe type: 3012/2, Kinematika, Switzerland) and extracted with methanol/chloroform $(1: 1 ; \mathrm{v} / \mathrm{v})$. The resultant monophasic extracts were evaporated to dryness, and the residues were dissolved in ethanol. The ethanolic solution was divided into 2 parts, one for alkaline hydrolysis with $5 \mathrm{M}$ potassium hydroxide $(\mathrm{KOH})$ and the other kept as a control; and they were placed in a water bath at $56^{\circ} \mathrm{C}$ for $1 \mathrm{~h}$. After cooling, the alkaline sample was neutralized with $5 \mathrm{M} \mathrm{HCl}$, and both it and the control were then partitioned with $\mathrm{H}_{2} \mathrm{O} / \mathrm{ethanol} / \mathrm{n}$-hexane $(1: 2: 4 ; \mathrm{v} / \mathrm{v} / \mathrm{v})$. The upper organic phase was evaporated to dryness, and the residue was dissolved in ethanol. The resultant ethanolic solution was analyzed by HPLC.

HPLC analysis for rice GGOH. The HPLC system (Tosoh Co., Tokyo) was composed of 2 pumps (DP-8020), a column reactor (CO-8020), and a UV detector equipped with a flow cell (UV8020). An aliquot of samples was injected onto a reverse-phase C18 column (Capcellpak C18, $4.6 \mathrm{~mm} \times 150 \mathrm{~mm}, 5 \mu \mathrm{m}$; Shiseido Fine Chemicals, Tokyo) with a mobile phase of methanol $/ 50 \mathrm{mM}$ ammonium acetate aqueous solution $(85: 15 ; \mathrm{v} / \mathrm{v})$ and eluted at a flow rate of $0.5 \mathrm{ml} / \mathrm{min}$ at $40^{\circ} \mathrm{C}$. The effluent was monitored by UV absorbance at $220 \mathrm{~nm}$. Chromatograms were recorded by a data processor (Chromatocorder 21, Tosoh Co., Tokyo). The concentration of GGPP in rice extracts was calculated from the peak area of GGOH after mild alkaline hydrolysis.

Enzyme assay.

Enzymatic dephosphorylation of GGPP. Bovine intestinal ALP (50 units/0.5 ml) (Sigma-Aldrich) was added to $50 \mathrm{mM}$ Tris- $\mathrm{HCl}$ buffer (pH 9.8) containing GGPP $(5-100 \mu \mathrm{M})$, and the reaction mixture was incubated for $1 \mathrm{~h}$ at $37^{\circ} \mathrm{C}$. The reaction was then stopped by chilling on ice, and the reaction mixture was diluted with 9 volumes of methanol. The resultant methanolic extract was analyzed by HPLC as described below.

Enzyme kinetics. Kinetic analyses were performed by using Lineweaver-Burk plots for each combination of enzyme and its substrate; i.e. ALP and GGPP, GGOH oxidase and GGOH, and GGal dehydrogenase and GGal. The data were expressed as means of 2 determinations from a representative experiment. Protein concentrations were determined by using a Bio-Rad Protein Assay kit. Cytochrome oxidase activity was measured as a marker enzyme for the mitochondrial fraction.

Enzymatic Oxidation of GGOH. The liver was removed from a male Wistar rat fasted overnight and homogenized in 2 volumes of $0.25 \mathrm{M}$ sucrose. Subcellular fractionation was conducted by differential centrifugation to obtain the mitochondrial (precipitated at $8,000 \times \mathrm{g}, 30 \mathrm{~min}$ ), microsomal (precipitated at $105,000 \times \mathrm{g}$, $90 \mathrm{~min}$ ) and cytosolic (supernatant at $105,000 \times \mathrm{g}, 90 \mathrm{~min}$ ) fractions. $200 \mu \mathrm{M}$ all-trans GGOH or $200 \mu \mathrm{M}$ GGal was incubated at $37^{\circ} \mathrm{C}$ with the whole homogenates or each subcellular fraction ( $0.5 \mathrm{mg}$ protein each) in $0.1 \mathrm{M}$ sodium phosphate buffer, $\mathrm{pH} 7.5$, containing $5 \mathrm{mM} \mathrm{MgCl} 2$ in the presence or absence of $5 \mathrm{mM}$ $\mathrm{NAD}^{+}$and/or $\mathrm{NADP}^{+}$in a final volume of $100 \mu \mathrm{l}$. The reaction was stopped by chilling on ice, and the reaction products were extracted twice with $200 \mu \mathrm{l}$ of diethylether each time. The diethylether was removed by nitrogen flushing, and the residue was treated with diazomethane at room temperature for $10 \mathrm{~min}$. The diazomethane-treated material was dissolved in $50 \mu \mathrm{l}$ of methanol, and the resultant methanolic solution $(20 \mu \mathrm{l})$ was injected onto a reversed-phase column of Zorbax ODS $(4.6 \times 150 \mathrm{~mm}$, Waters, MA) with a mobile phase of methanol/water $(85: 15 ; \mathrm{v} / \mathrm{v})$ at a flow rate of $0.5 \mathrm{ml} / \mathrm{min}$. UV absorption of compounds eluted was monitored at a fixed wave length of $250 \mathrm{~nm}$.

\section{Results}

GGPP and GGOH in rice. Fig. 2 shows a representative HPLC analysis of GGPP in lipid extracts from Kinuhikari polished rice. GGPP was not eluted in untreated rice extracts (Fig. 2a); however, after being subjected to mild alkaline hydrolysis with $\mathrm{KOH}$, a prominent peak corresponding to authentic GGOH was detected clearly (Fig. 2b), indicating the presence of GGPP in polished rice (polished to $88 \%$ ). Even in untreated extracts, a small peak corresponding to authentic GGOH was detected, indicating that a small amount of free GGOH might be contained in polished rice. In contrast, the GGA concentration in rice was under the minimum detection limit either by this HPLC analysis or by LC/MS analysis.

From each peak area of GGOH in 2 separate runs of HPLC before and after $\mathrm{KOH}$ treatment, the amounts of $\mathrm{GGOH}$ and GGOH + GGPP were calculated, respectively. Table 1 shows the estimated contents of GGPP and GGOH in brown and $87-89 \%$ polished rice of four cultivars of Japonica rice. GGPP contents in rice ranged from 0 to $2.9 \mu \mathrm{g} / \mathrm{g}$ with large variation depending on the rice cultivars. The GGPP content in Koshihikari rice was under the minimum detection limit $(0.02 \mu \mathrm{g} / \mathrm{g})$ in both brown and polished rice; whereas in Karinomai rice a relatively large amount of GGPP $(2.08 \mu \mathrm{g} / \mathrm{g})$ was detected only in the brown rice, indicating that GGPP was present in the outer layers of the rice seeds (pericarp, tegmen or aleurone layer). The content of GGPP in Hinohikari polished rice $(0.73 \mu \mathrm{g} / \mathrm{g})$ was slightly higher than that in brown rice $(0.70 \mu \mathrm{g} / \mathrm{g})$, indicating that GGPP in Hinohikari may tend to be produced to a greater extent in the endosperm than in the outer layers. Further, the amount of GGPP in Kinuhikari brown rice $(2.88 \mu \mathrm{g} / \mathrm{g})$ was the highest and 3 times greater than that in polished rice $(1.04 \mu \mathrm{g} / \mathrm{g})$, indicating that GGPP may be produced in both the endosperm and the outer layers of this cultivar.

As a whole, GGPP distribution in rice could likely be the following: outer layers in Karinomai, endosperm in Hinohikari, and both of them in Kinuhikari. The data thus suggest that the content and distribution of GGPP varies depending on the type of cultivar. Further study will be needed on other rice variants, their storage conditions, and so forth. The amount of GGPP (0.73-1.04 $\mu \mathrm{g} / \mathrm{g})$ in polished rice analyzed here approximated that of $\beta$ carotene $(1.6 \mu \mathrm{g} / \mathrm{g})$ produced in genetically modified rice reported by Ye et $a l .^{(18)}$

\section{Enzymatic conversion from geranylgeranyl diphosphate to geranylgeranoic Acid.}

Desphosphorylation of GGPP by ALP. GGPP was definitely found in edible parts of some rice cultivars. Accordingly, we next examined the possibility that GGPP from the diet could be metabolized to GGA in the human body. In converting GGPP into GGA, at the first step the terminal pyrophosphate group of GGPP must be removed. Here we confirmed the possibility of this happening in vivo by using bovine intestinal ALP, which is known to have a relatively low-substrate specificity in gut mucosa. As clearly shown by HPLC analysis in Fig. $2 \mathrm{c}$ and d, GGPP was converted into GGOH by ALP; and the conversion rate linearly increased in a time-dependent fashion for 40 min (data not shown).

Since we found that ALP used GGPP as a substrate, kinetic analysis was performed on the assumption that "GGPP = $\mathrm{GGOH}+2 \mathrm{Pi}$ " was a set of enzymatic reactions of monoesterhydrolysis. The ALP assay was carried out by varying the GGPP concentration $(5-100 \mu \mathrm{M})$ and keeping the concentration of ALP $(0.01$ units $/ 50 \mu \mathrm{l})$ constant to determine the maximum reaction velocity $\mathrm{V}_{\max }$ and apparent $K_{\mathrm{m}}$. The apparent $K_{\mathrm{m}}$ of ALP was found to be $46.1 \mu \mathrm{M}$ for GGPP. This $K_{\mathrm{m}}$ is relatively low among those values for the known substrates $(1-10 \mathrm{mM})$ so far reported;(22) and thus GGPP would supposedly have a relatively high affinity for ALP. For example, the $K_{\mathrm{m}}$ for pNPP, which is a synthetic substrate used to measure the enzyme activity of ALP, is known 


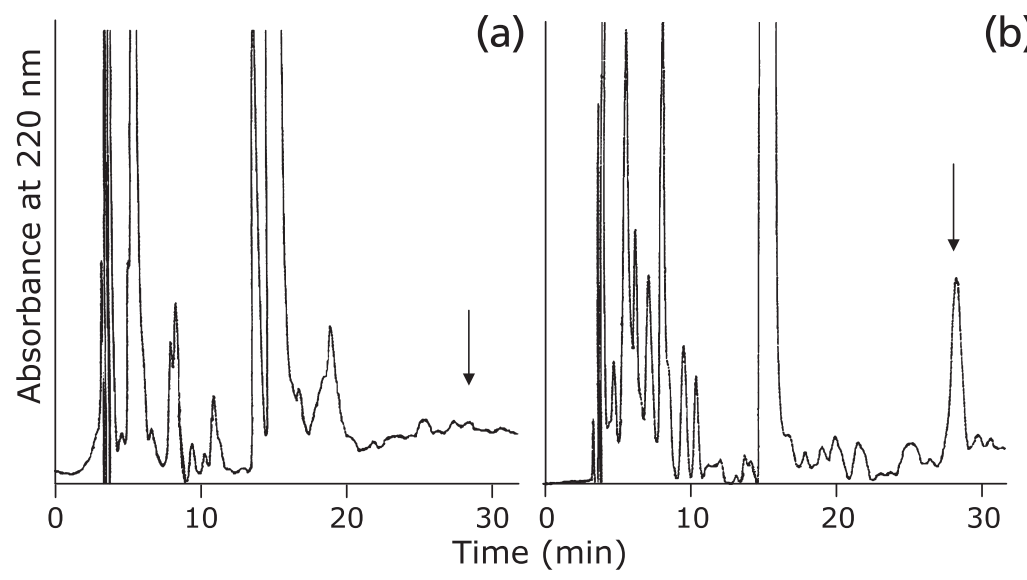

(b)

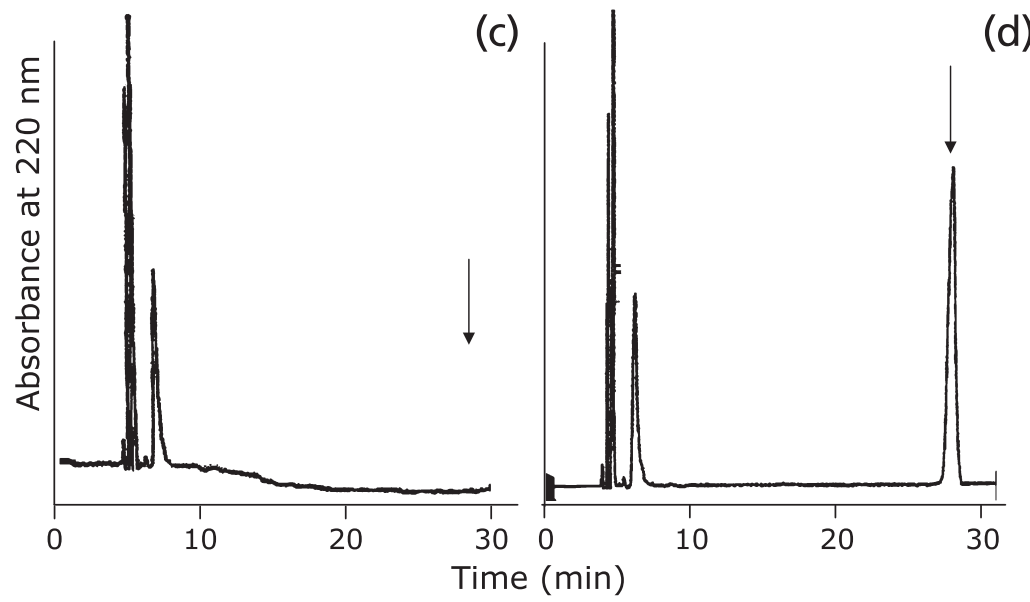

Fig. 2. Dephosphorylation of geranylgeranyl diphosphate (GGPP). Reversed-phase HPLC chromatogram of the lipid extracts from Kinuhikari polished rice before (a) and after (b) $\mathrm{KOH}$ treatment, and from $100 \mu \mathrm{M}$ GGPP solution in $50 \mathrm{mM}$ Tris-HCl buffer (pH 9.8) without (c) or with (d) bovine ALP. Details of the experimental procedure were described in Materials and Methods. Arrows indicate the elution position of authentic GGOH.

Table 1. Concentrations of GGPP and GGOH in rice

\begin{tabular}{|c|c|c|c|c|c|c|}
\hline \multirow{2}{*}{ rice cultivars } & \multicolumn{2}{|c|}{ GGPP $(\mu \mathrm{g} / \mathrm{g})$} & \multicolumn{2}{|c|}{$\mathrm{GGOH}(\mu \mathrm{g} / \mathrm{g})$} & \multicolumn{2}{|c|}{$\mathrm{GGPP}+\mathrm{GGOH}(\mu \mathrm{g} / \mathrm{g})$} \\
\hline & brown & polished & brown & polished & brown & polished \\
\hline Koshihikari & - & - & - & - & - & - \\
\hline Kinuhikari & 2.88 & 1.04 & 0.09 & 0.23 & 2.97 & 1.26 \\
\hline Hinohikari & 0.70 & 0.73 & - & - & 0.70 & 0.73 \\
\hline Karinomai & 2.08 & - & - & - & 2.08 & - \\
\hline
\end{tabular}

Values are means of 2 determinations. GGPP, geranylgeranyl diphosphate; GGOH, geranylgeraniol. The grains of polished rice were polished to $87-$ $89 \%$ of their original weight. —: under detection limit $(0.02 \mu \mathrm{g} / \mathrm{g})$.

to be approximately $0.9 \mathrm{mM}$.

The GGPP contents in rice analyzed here were $0.7-3.0 \mu \mathrm{g} / \mathrm{g}$ (1-6 mM), which may provide adequate concentrations for the enzyme activity. The efficiency of hydrolysis of GGPP to GGOH in the small intestine, however, depends on other factors such as ALP concentration, the solubility of GGPP in the intestinal contents, and the time that GGPP remains in the intestine. Further in vivo study will be needed to establish the intestinal dephosphorylation of exogenously supplied GGPP.

There have been some reports of intracellular pyrophosphatase activities for prenyl pyrophosphate (EC3.1.7.1) in mammals. The apparent $K_{\mathrm{m}}$ of pyrophosphatase from rat testicle for IPP was 4-10 mM; and that for FPP, 0.7-1.2 mM. ${ }^{(23)}$ Bansal and Vaidya ${ }^{(24)}$ determined geranylgeranyl pyrophosphate-specific pyrophosphatase (GGPPase) activity in rat liver microsomes. This GGPPase had specificity and high affinity for GGPP and converted GGPP to GGOH with the liberation of pyrophosphate. The microsomal GGPPase had a $K_{\mathrm{m}}$ of $12 \mu \mathrm{M}$ for GGPP and did not hydrolyze a FPP substrate. The $K_{\mathrm{m}}$ of bovine intestinal ALP for GGPP, measured in this study, was approximately that of rodent-specific GGPPase rather than those values of unspecific pyrophosphatases for prenylpyrophosphate. Accordingly, the intestinal ALP would supposedly hydrolyze GGPP to GGOH efficiently. Next, it was intriguing for us to examine the possibility that GGA might be enzymatically and metabolically synthesized from GGOH in the liver. 


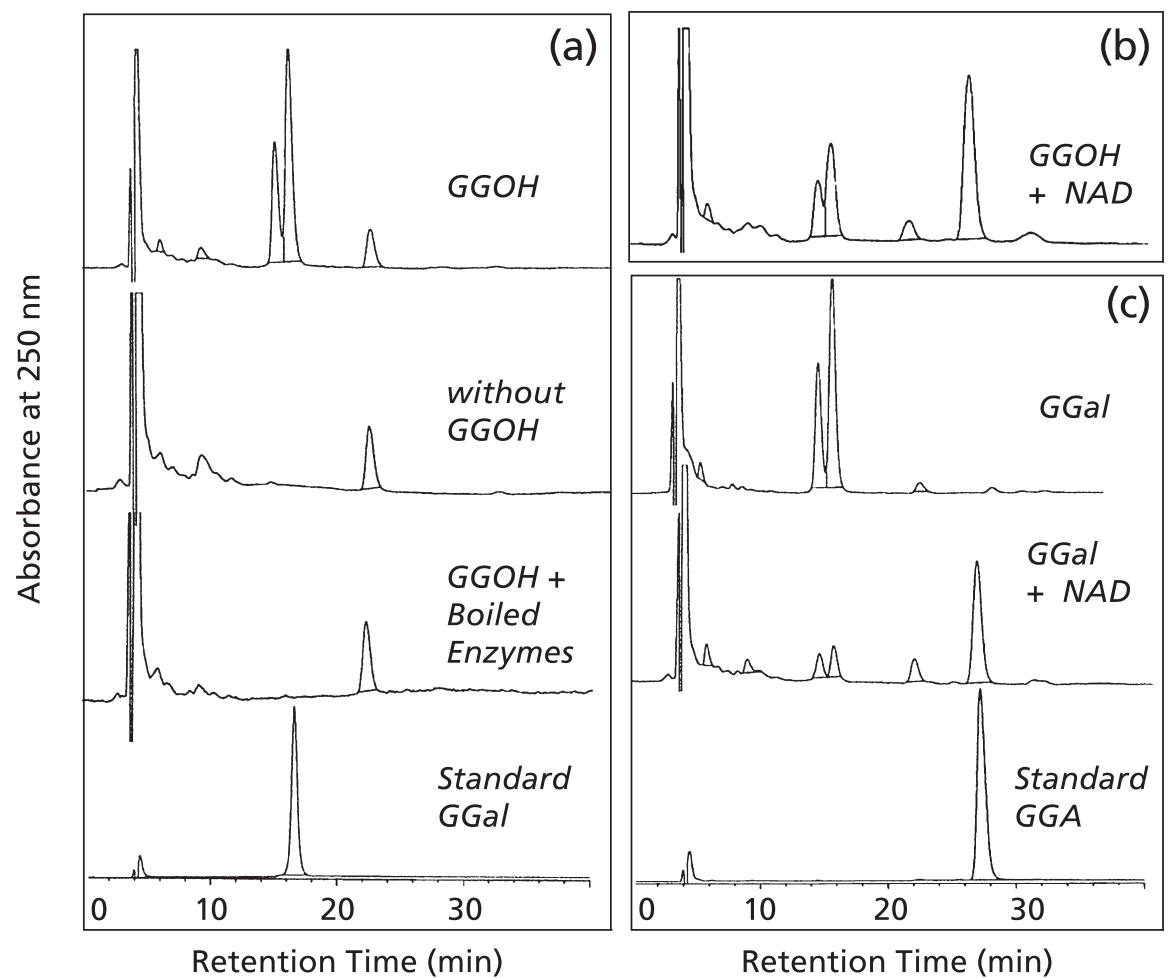

Fig. 3. Enzymatic conversion of geranylgeraniol (GGOH) to geranylgeranoic acid (GGA) via geranylgeraniol (GGal) in rat liver. Shown are HPLC profiles of the diazomethane-treated ether extracts from the reaction mixtures of rat liver homogenates (0.5 mg protein) with or without $200 \mu \mathrm{M}$ GGOH (a), with $200 \mu \mathrm{M} \mathrm{GGOH}$ and $5 \mathrm{mM} \mathrm{NAD}^{+}$(b) and with $200 \mu \mathrm{M} \mathrm{GGal}$ in the absence or presence of $\mathrm{NAD}^{+}$(c). The elution profiles of diazomethane-treated authentic GGal and GGA are shown in the lowest chromatogram in panels a and c, respectively.

Enzymatic oxidation of GGOH to GGal. When GGOH was incubated with rat liver homogenates, a prominent peak of GGal was produced (Fig. 3a). The conversion of GGOH to GGal was dependent on the amount of the homogenate proteins and sensitive to heat inactivation $\left(95^{\circ} \mathrm{C}, 5 \mathrm{~min}\right)$, as shown in Fig. 3a. Addition of $\mathrm{NAD}^{+}$and/or $\mathrm{NADP}^{+}$to the reaction mixture decreased the amount of GGal produced and instead a new peak, which corresponded to GGA methylester or methyl geranylgeranoate, appeared on HPLC (Fig. 3b). Furthermore a pooled fraction from the GGal peak (16.5-17.5 min) was converted to GGA by rat liver homogenates in the presence of $5 \mathrm{mM} \mathrm{NAD}^{+}$(data not shown). This finding clearly shows that GGA was undoubtedly synthesized from GGOH by at least 2 enzymatic reactions consisting of nucleotide-independent and pyridine nucleotide-dependent processes. A peak slightly preceding the GGal peak (Fig. 3a) has not yet been identified, but when it was incubated with $\mathrm{NAD}^{+}$in rat liver homogenates and treated with diazomethane, it was converted to a peak corresponding to GGA methylester (data not shown).

In the absence of $\mathrm{NAD}^{+}$or $\mathrm{NADP}^{+}$, we can focus attention on the initial reaction. Since the proton acceptor from GGOH during the initial reaction is still unidentified, we tentatively name this enzyme "GGOH oxidase" instead of GGOH dehydrogenase. As shown in Table 2, the GGOH-oxidizing activity was mainly localized in the mitochondrial fractions; and no activity was found in the soluble fraction. A Lineweaver-Burk plot with the mitochondrial enzyme gave a $K_{\mathrm{m}}$ of $92.9 \mu \mathrm{M}$ for $\mathrm{GGOH}$, which was relatively higher than the reported $K_{\mathrm{m}}$ value $(12 \mu \mathrm{M})$ of GGPPase for GGPP. ${ }^{(24)}$ The $V_{\max }(1.58 \mathrm{nmol} / \mathrm{mg}$ prot $/ \mathrm{h})$ of the mitochondrial enzyme was lower than that $(840 \mathrm{nmol} / \mathrm{mg} \operatorname{prot} / \mathrm{h})$ of microsomal GGPPase.(24) Hence, GGOH oxidase may create a rate-limiting step in GGA synthesis.
Table 2. Subcellular distributions of GGOH-oxidizing activity and GGal dehydrogenase activity in rat liver

\begin{tabular}{ccc}
\hline \multirow{2}{*}{ subcellular fraction } & GGOH oxidase & GGal dehydrogenase \\
\cline { 2 - 3 } & \multicolumn{2}{c}{$\mathrm{nmol} / \mathrm{mg} \mathrm{prot} / \mathrm{h}$} \\
\hline post nuclear & $0.231 \pm 0.01$ & $5.32 \pm 0.25$ \\
mitochondria & $0.792 \pm 0.03$ & $13.10 \pm 0.62$ \\
microsomes & $0.348 \pm 0.01$ & $6.10 \pm 0.36$ \\
cytosol & $<0.03$ & $5.20 \pm 0.21$
\end{tabular}

Values are means \pm SD of 3 determinations. GGOH, geranylgeraniol; GGal, geranylgeranial.

Enzymatic conversion of GGal to GGA. By using synthetic GGal, we focused on the second reaction. GGal was quite stable at $37^{\circ} \mathrm{C}$ in rat liver homogenates without $\mathrm{NAD}^{+}$or $\mathrm{NADP}^{+}$, except that a peak preceding GGal emerged on HPLC after incubation with rat liver homogenates (Fig. 3c). But, as mentioned previously the same preceding peak came out during the conversion from GGOH to GGal (Fig. 3a).

The conversion of GGal to GGA by rat liver homogenates was absolutely dependent on exogenously added $\mathrm{NAD}^{+}$or $\mathrm{NADP}^{+}$ (Fig. 3c). A half maximal rate of the reaction was obtained with $2.3 \mathrm{mM} \mathrm{NAD}^{+}$or $1.6 \mathrm{mM} \mathrm{NADP}^{+}$. Inasmuch as the proton acceptor was identified as $\mathrm{NAD}^{+}$(or $\mathrm{NADP}^{+}$), we named this enzyme "GGal dehydrogenase". The GGal dehydrogenase activity was localized mainly in the mitochondrial fraction, though significant activity was also found in both the microsomal and cytosolic fractions (Table 2). The $K_{\mathrm{m}}$ of the mitochondrial GGal dehydrogenase was $27.5 \mu \mathrm{M}$ for GGal, and the $\mathrm{V}_{\max }$ of the mitochondrial enzyme was $13.1 \mathrm{nmol} / \mathrm{mg}$ prot/h (Table 2). Although 
the substrate specificity of the mitochondrial dehydrogenase was not investigated, these kinetic parameters of the enzyme show high affinity for GGal and an efficient rate to catalyze the conversion of GGal to GGA in hepatocytes. At least in cell-free systems, we could thus far demonstrate that GGA could be enzymatically synthesized from GGOH, the dephosphorylation product of GGPP.

\section{Discussion}

As the first main finding of the present study, we definitely confirmed the presence of GGPP in edible parts of rice. Then we presented several lines of evidence to demonstrate that the exogenous GGPP (or GGPP in food) could be converted to GGA by intestinal and hepatic enzymes (Fig. 4).

Our focus was directed at the potential ability of rice endosperm to produce and accumulate a relatively large amount of $\beta$-carotene $\left(1.6-6.0 \mu \mathrm{g} / \mathrm{g}\right.$ of dry rice). ${ }^{(20,25)}$ It has been suggested that ordinary (non-transgenic) polished rice may contain a considerable amount of GGPP. However, no particular attention has been given to the role of GGPP as a lipid in rice excluding that of a precursor of $\beta$ carotene. From a simple calculation based on our present data on polished rice, we estimate that Japanese individuals may consume a substantial amount $(\sim 500 \mu \mathrm{g} /$ day $)$ of GGPP through a staple food, i.e., rice. Since a significant amount of GGPP and its derivatives were detected in rice, our next question was whether or not GGA could be enzymatically synthesized from GGPP in the human body.

Besides the exogenous GGPP, our cells may be inherently familiar with "endogenous" GGPP. In 1983, all-trans GGPP synthetase was found in pig liver. ${ }^{(26)}$ This enzyme catalyzes 2 consecutive isoprenyl-transfer reactions, one from GPP to FPP

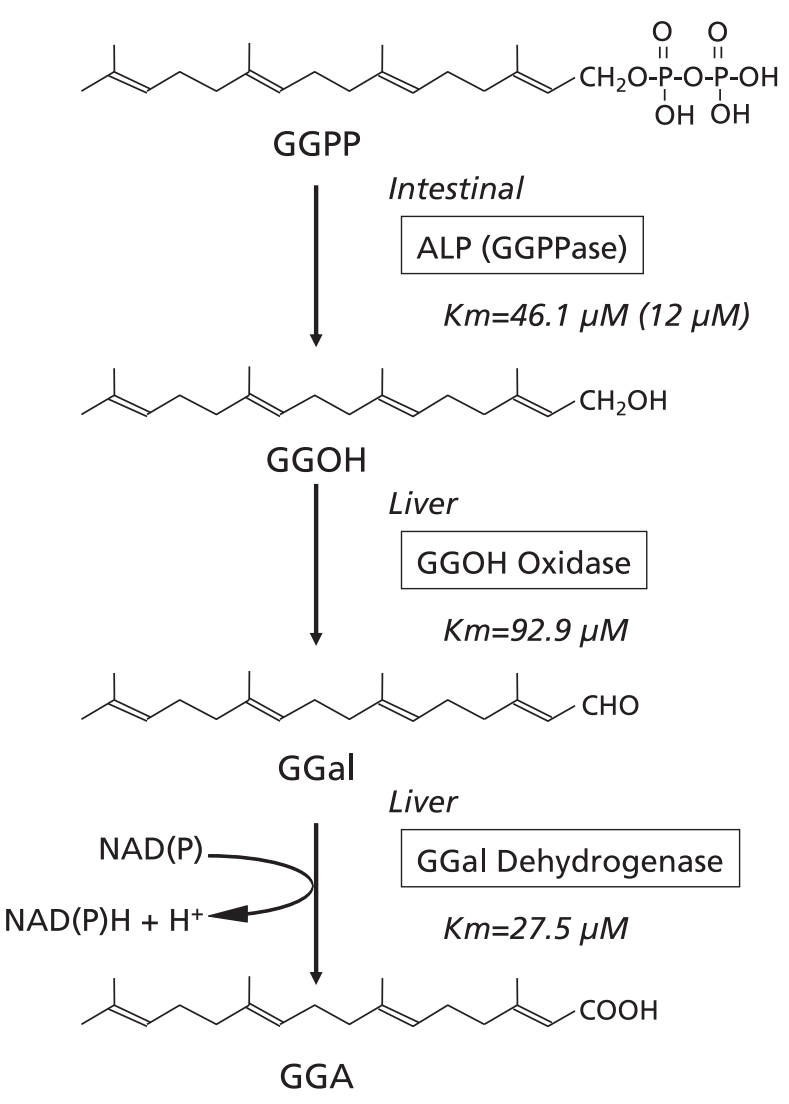

Fig. 4. Proposed enzymatic conversion from geranylgeranyl diphosphate (GGPP) to geranylgeranoic acid (GGA). and the other from FPP to GGPP. Although the metabolic fate or biologic roles of all-trans GGPP were not addressed in that 1983 study, endogenous GGPP is now well established as one of the isoprenoid donors for protein isoprenylations. ${ }^{(27-29)}$ But, as Dallner's group pointed out, GGPP synthetase activity is almost 100-times higher than protein-geranylgeranyl transferase activity in rat brain. (30) GGPP may have other metabolic pathways in addition to protein-geranylgeranylation or all-trans GGPP may be required for other, as yet unidentified, cellular processes.

Bansal and Vaidya have found geranylgeranyl pyrophosphatase (GGPPase) activity in rat liver microsomes. ${ }^{(24)}$ The enzyme was the most active at physiologic $\mathrm{pH}$ and highly specific for GGPP, and did not hydrolyze FPP or other allyl pyrophosphates. Once GGOH is produced through dephosphorylation of GGPP by the specific pyrophosphatase in hepatocytes, it seems reasonable to assume that the non-specific fatty alcohol dehydrogenase/fatty aldehyde dehydrogenase system might produce GGA through the intermediate GGal.

At the beginning of the present study, we expected that some non-specific alcohol dehydrogenase ${ }^{(31)}$ might be involved in the production of GGal. But, this was not the case, because the oxidation of GGOH by a hepatic enzyme was independent of exogenous pyridine-nucleotides and the cytosolic fraction contained scarce activity for GGOH oxidation. Usually, non-specific alcohol dehydrogenase is dependent on $\mathrm{NAD}^{+}$and resides mainly in the soluble fraction. Inasmuch as the proton acceptor from GGOH has not yet been identified, the mitochondrial GGOH-oxidizing enzyme cannot be called "GGOH dehydrogenase".

In the course of the biosynthesis of juvenile hormone, an insect $\mathrm{FOH}$ dehydrogenase catalyzes the conversion of $\mathrm{FOH}$ to farnesyl aldehyde; and the activity of this enzyme is apparently independent of exogenous $\mathrm{NAD}^{+}$or $\mathrm{NADP}^{+}$, although the addition of $\mathrm{NAD}^{+}$stimulates the oxidation of FOH to farnesoic acid. ${ }^{(32)}$ This insect farnesoic acid-generating system is somewhat similar to the mammalian GGOH oxidation system found in this study. Furthermore, the insect FOH dehydrogenase was later proved not to be a dehydrogenase but an oxidase with a cofactor of FAD. ${ }^{(33,34)}$ We should test the FAD dependence of rodent GGOH oxidase and investigate the evolutionary relationship between insect FOH dehydrogenase and the rodent GGOH-oxidizing enzyme.

Recently, a fascinating enzyme called prenylcysteine lyase, a lysosomal monooxygenase, was reported to be potentially involved in the production of GGal as well as farnesal in mammalian hepatocytes. ${ }^{(35-38)}$ This lysosomal enzyme acts on a substrate of geranylgeranylcysteine, which is a degradation product of cellular geranylgeranylated proteins, to liberate a product of GGal and transfer protons to molecular oxygen. One cannot exclude the possibility that this lysosomal enzyme may well be active toward GGOH to generate GGal.

As for GGal dehydrogenase, we were unable to distinguish the enzyme from NAD $(\mathrm{P})^{+}$-dependent aldehyde dehydrogenases with common structural and functional features that catalyze the oxidation of a broad spectrum of aliphatic and aromatic aldehydes. ${ }^{(39)}$ Based on amino acid sequence analysis, 3 major classes of mammalian aldehyde dehydrogenases have been identified: classes 1 and 3 are cytosolic and class 2 is mitochondrial. ${ }^{(40)}$ In addition, human cDNA for microsomal fatty aldehyde dehydrogenase has been cloned, and this hepatic enzyme is active toward a variety of saturated and unsaturated aliphatic aldehydes between 6 and 24 carbons in length. ${ }^{(41)}$ We found significant GGal dehydrogenase activity in the mitochondrial and microsomal fractions as well as in the cytosolic fraction, where little, if any, aldehyde dehydrogenase activity is found in normal rat liver, ${ }^{(41)}$ suggesting that the cytosolic GGal dehydrogenase may be unique and may not be common aldehyde dehydrogenase.

In any case, to establish the enzymatic reactions from GGOH to GGA in rat liver, we absolutely need more detailed biochemical and molecular biological characterizations of the enzymatic con- 
version from GGOH to GGA found in the present study (Fig. 4).

In summary, evidence is provided in support of possible exogenous precursors for GGA such as GGPP and GGOH in rice. Further, we have provided biochemical evidence for the enzymatic production of GGA from GGOH or GGPP in mammalian cells. More detailed molecular biological analysis of eukaryotic GGAgenerating systems should shed more light on the biology of GGA. $^{(42)}$

\section{Acknowledgments}

This project was supported in part by a grant-in-aid from the Japanese Rice Stable Supply Support Organization.

\section{References}

1 Muto Y, Moriwaki H, Omori M. In vitro binding affinity of novel synthetic polyprenoids (polyprenoic acids) to cellular retinoid-binding proteins. Gann 1981; 72: 974-977.

2 Araki H, Shidoji Y, Yamada Y, Moriwaki H, Muto Y. Retinoid agonist activities of synthetic geranyl geranoic acid derivatives. Biochem Biophys Res Commun 1995; 209: 66-72.

3 Yamada Y, Shidoji Y, Fukutomi Y, and et al. Positive and negative regulations of albumin gene expression by retinoids in human hepatoma cell lines. Mol Carcinog 1994; 10: 151-158.

4 Fukutomi Y, Omori M, Muto Y, Ninomiya M, Okuno M, Moriwaki H. Inhibitory effects of acyclic retinoid (polyprenoic acid) and its hydroxy derivative on cell growth and on secretion of alpha-fetoprotein in human hepatoma-derived cell line (PLC/PRF/5). Jpn J Cancer Res 1990; 81: 12811285.

5 Muto Y, Moriwaki H. Antitumor activity of vitamin A and its derivatives. $J$ Natl Cancer Inst 1984; 73: 1389-1393.

6 Muto Y, Moriwaki H, Ninomiya M, and et al. Prevention of second primary tumors by an acyclic retinoid, polyprenoic acid, in patients with hepatocellular carcinoma. Hepatoma Prevention Study Group. N Engl J Med 1996; 334: $1561-1567$.

7 Muto Y, Moriwaki H, Saito A. Prevention of second primary tumors by an acyclic retinoid in patients with hepatocellular carcinoma. $N$ Engl $\mathrm{J}$ Med 1999; 340: 1046-1047.

8 Shidoji Y, Nakamura N, Moriwaki H, Muto Y. Rapid loss in the mitochondrial membrane potential during geranylgeranoic acid-induced apoptosis. Biochem Biophys Res Commun 1997; 230: 58-63.

9 Nakamura N, Shidoji Y, Moriwaki H, Muto Y. Apoptosis in human hepatoma cell line induced by 4,5-didehydro geranylgeranoic acid (acyclic retinoid) via down-regulation of transforming growth factor-alpha. Biochem Biophys Res Commun 1996; 219: 100-104.

10 Sacchettini JC, Poulter CD. Creating isoprenoid diversity. Science 1997; 277: 1788-1789.

11 Lichtenthaler HK. Non-mevalonate isoprenoid biosynthesis: enzymes, genes and inhibitors. Biochem Soc Trans 2000; 28: 785-789.

12 Seybold SJ, Tittiger C. Biochemistry and molecular biology of de novo isoprenoid pheromone production in the Scolytidae. Annu Rev Entomol 2003; 48: $425-453$.

13 Rohmer M. The discovery of a mevalonate-independent pathway for isoprenoid biosynthesis in bacteria, algae and higher plants. Nat Prod Rep 1999; 16: $565-574$.

14 Hamberger B, Bohlmann J. Cytochrome P450 mono-oxygenases in conifer genomes: discovery of members of the terpenoid oxygenase superfamily in spruce and pine. Biochem Soc Trans 2006; 34: 1209-1214.

15 Joo JH, Jetten AM. Molecular mechanisms involved in farnesol-induced apoptosis. Cancer Lett 2010; 287: 123-135.

16 Holstein SA, Hohl RJ. Isoprenoids: remarkable diversity of form and function. Lipids 2004; 39: 293-309.

17 Shidoji Y, Ogawa H. Natural occurrence of cancer-preventive geranylgeranoic acid in medicinal herbs. J Lipid Res 2004; 45: 1092-1103.

18 Ye X, Al-Babili S, Klöti A, and et al. Engineering the provitamin A (betacarotene) biosynthetic pathway into (carotenoid-free) rice endosperm. Science 2000; 287: 303-305.

19 Burkhardt PK, Beyer P, Wünn J, and et al. Transgenic rice (Oryza sativa) endosperm expressing daffodil (Narcissus pseudonarcissus) phytoene synthase

\section{Abbreviations}

\section{DMAPP dimethylallyl diphosphate}

FOH farnesol

FPP farnesyl diphosphate

GGA geranylgeranoic acid

GGal geranylgeranial

GGOH geranylgeraniol

GGPP geranylgeranyl diphosphate

GPP geranyl diphosphate

IPP isopentenyl diphosphate accumulates phytoene, a key intermediate of provitamin A biosynthesis. Plant $J$ 1997; 11: 1071-1078.

20 Beyer P, Al-Babili S, Ye X, and et al. Golden Rice: introducing the betacarotene biosynthesis pathway into rice endosperm by genetic engineering to defeat vitamin A deficiency. J Nutr 2002; 132: S506-S510.

21 Yeh KY, Yeh M, Glass J. Expression of intestinal brush-border membrane hydrolases and ferritin after segmental ischemia-reperfusion in rats. $\mathrm{Am} \mathrm{J}$ Physiol 1998; 275: G572-G583.

22 Daniel H, Binninger E, Rehner G. Hydrolysis of FMN and FAD by alkaline phosphatase of the intestinal brush-border membrane. Int J Vitam Nutr Res 1983; 53: 109-114.

23 Tsai SC, Gaylor JL. Testicular sterols. V. Preparation and partial purification of a microsomal prenol pyrophosphate pyrophosphohydrolase. $J$ Biol Chem 1966; 241: 4043-4050.

24 Bansal VS, Vaidya S. Characterization of two distinct allyl pyrophosphatase activities from rat liver microsomes. Arch Biochem Biophys 1994; 315: 393399

25 Al-Babili S, Beyer P. Golden rice-five years on the road-five years to go? Trends Plant Sci 2005; 10: 565-573.

26 Ishii K, Sagami H, Ogura K. Decaprenyl pyrophosphate synthetase from mitochondria of pig liver. Biochem Biophys Res Commun 1983; 116: 500 506.

27 Maltese WA. Posttranslational modification of proteins by isoprenoids in mammalian cells. FASEB J 1990; 4: 3319-3328.

28 Kuzuguchi T, Morita Y, Sagami I, Sagami H, Ogura K. Human geranylgeranyl diphosphate synthase. cDNA cloning and expression. J Biol Chem 1999; 274 5888-5894.

29 Hannoush RN, Sun J. The chemical toolbox for monitoring protein fatty acylation and prenylation. Nat Chem Biol 2010; 6: 498-506.

30 Grünler J, Ericsson J, Dallner G. Branch-point reactions in the biosynthesis of cholesterol, dolichol, ubiquinone and prenylated proteins. Biochim Biophys Acta 1994; 1212: 259-277.

31 Kedishvili NY, Bosron WF, Stone CL, and et al. Expression and kinetic characterization of recombinant human stomach alcohol dehydrogenase. Active-site amino acid sequence explains substrate specificity compared with liver isozymes. J Biol Chem 1995; 270: 3625-3630.

32 Baker FC, Mauchamp B, Tsai LW, Schooley DA. Farnesol and farnesal dehydrogenase(s) in corpora allata of the tobacco hornworm moth, Manduca sexta. J Lipid Res 1983; 24: 1586-1594.

33 Sperry AE, Sen SE. Farnesol oxidation in insects: evidence that the biosynthesis of insect juvenile hormone is mediated by a specific alcohol oxidase. Insect Biochem Mol Biol 2001; 31: 171-178.

34 Sen SE, Sperry AE, Childress M, Hannemann DE. Juvenile hormone biosynthesis in moths: synthesis and evaluation of farnesol homologs as alternate substrates of farnesol oxidase. Insect Biochem Mol Biol 2003; 33: 601-607.

35 Tschantz WR, Zhang L, Casey PJ. Cloning, expression, and cellular localization of a human prenylcysteine lyase. J Biol Chem 1999; 274: 35802-35808.

36 Tschantz WR, Digits JA, Pyun HJ, Coats RM, Casey PJ. Lysosomal prenylcycsteine lyase is a FAD-dependent thioether oxidase. J Biol Chem 2001; 276: 2321-2324.

37 Lu JY, Hofmann SL. Lysosomal metabolism of lipid-modified proteins. $J$ Lipid Res 2006; 47: 1352-1357.

38 Banfi C, Brioschi M, Barcella S, and et al. Proteomic analysis of human lowdensity lipoprotein reveals the presence of prenylcysteine lyase, a hydrogen 
peroxide-generating enzyme. Proteomics 2009; 9: 1344-1352.

39 Lindahl R. Aldehyde dehydrogenases and their role in carcinogenesis. Crit Rev Biochem Mol Biol 1992; 27: 283-335.

40 De Laurenzi V, Rogers GR, Hamrock DJ, and et al. Sjögren-Larsson syndrome is caused by mutations in the fatty aldehyde dehydrogenase gene. Nat Genet 1996; 12: 52-57.
41 Lindahl R. Subcellular distribution and properties of aldehyde dehydrogenase from 2-acetylaminofluorene-induced rat hepatomas. Biochem J 1979; 183: 55-64.

42 Wang X, Wu J, Shidoji Y, and et al. Effects of geranylgeranoic acid in bone: induction of osteoblast differentiation and inhibition of osteoclast formation. $J$ Bone Miner Res 2002; 17: 91-100. 